\title{
Abstract and Effector-Selective Decision Signals Exhibit Qualitatively Distinct Dynamics before Delayed Perceptual Reports
}

\author{
Deirdre M. Twomey, ${ }^{1}$ Simon P. Kelly, ${ }^{2,3}$ and Redmond G. 0'Connell ${ }^{1}$ \\ ${ }^{1}$ Trinity College Institute of Neuroscience and School of Psychology, Trinity College Dublin, Dublin 2, Ireland, ${ }^{2}$ Department of Biomedical Engineering, City \\ College of the City University of New York, New York, New York 10031, and ${ }^{3}$ School of Electrical and Electronic Engineering, University College Dublin, \\ Dublin 4, Ireland
}

Electrophysiological research has isolated neural signatures of decision formation in a variety of brain regions. Studies in rodents and monkeys have focused primarily on effector-selective signals that translate the emerging decision into a specific motor plan, but, more recently, research on the human brain has identified an abstract signature of evidence accumulation that does not appear to play any direct role in action preparation. The functional dissociations between these distinct signal types have only begun to be characterized, and their dynamics during decisions with deferred actions with or without foreknowledge of stimulus-effector mapping, a commonly studied task scenario in single-unit and functional imaging investigations, have not been established. Here we traced the dynamics of distinct abstract and effector-selective decision signals in the form of the broad-band centro-parietal positivity (CPP) and limb-selective $\beta$-band $(8-16$ and $18-30 \mathrm{~Hz})$ EEG activity, respectively, during delayed-reported motion direction decisions with and without foreknowledge of direction-response mapping. With foreknowledge, the CPP and $\beta$-band signals exhibited a similar gradual build-up following evidence onset, but whereas choice-predictive $\beta$-band activity persisted up until the delayed response, the CPP dropped toward baseline after peaking. Without foreknowledge, the CPP exhibited identical dynamics, whereas choice-selective $\beta$-band activity was eliminated. These findings highlight qualitative functional distinctions between effector-selective and abstract decision signals and are of relevance to the assumptions founding functional neuroimaging investigations of decision-making.

Key words: EEG; perceptual decision-making

Significance Statement

Neural signatures of evidence accumulation have been isolated in numerous brain regions. Although animal neurophysiology has largely concentrated on effector-selective decision signals that translate the emerging decision into a specific motor plan, recent research on the human brain has isolated abstract neural signatures of decision formation that are independent of specific sensory and motor requirements. Here, we examine the functional distinctions between the two distinct classes of decision variable signal during decisions with deferred actions with and without foreknowledge of stimulus-effector mapping. We find salient distinctions in the dynamics of abstract versus effector-selective decision signals in the human brain, in terms of sustainment through response delays and contingency on foreknowledge of stimulus-response mapping.

\section{Introduction}

Single-unit recordings in rodents and monkeys have identified neural populations whose spiking activity integrates sensory evi- dence during perceptual decision formation and triggers the decision-reporting action upon reaching a threshold level (Shadlen and Kiani, 2013). More recently, human electrophysi-

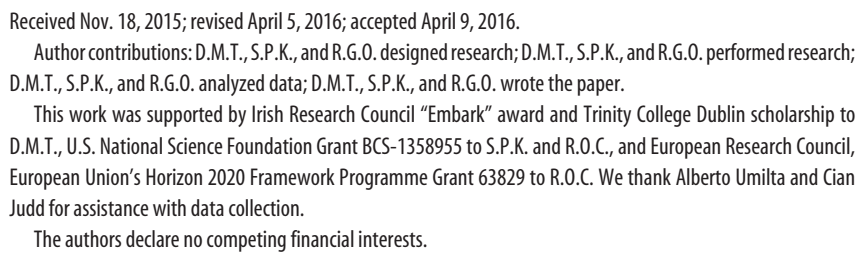

The authors declare no competing financial interests.

This is an Open Access article distributed under the terms of the Creative Commons Attribution License Creative Commons Attribution 4.0 International, which permits unrestricted use, distribution and reproduction in any medium provided that the original work is properly attributed. 


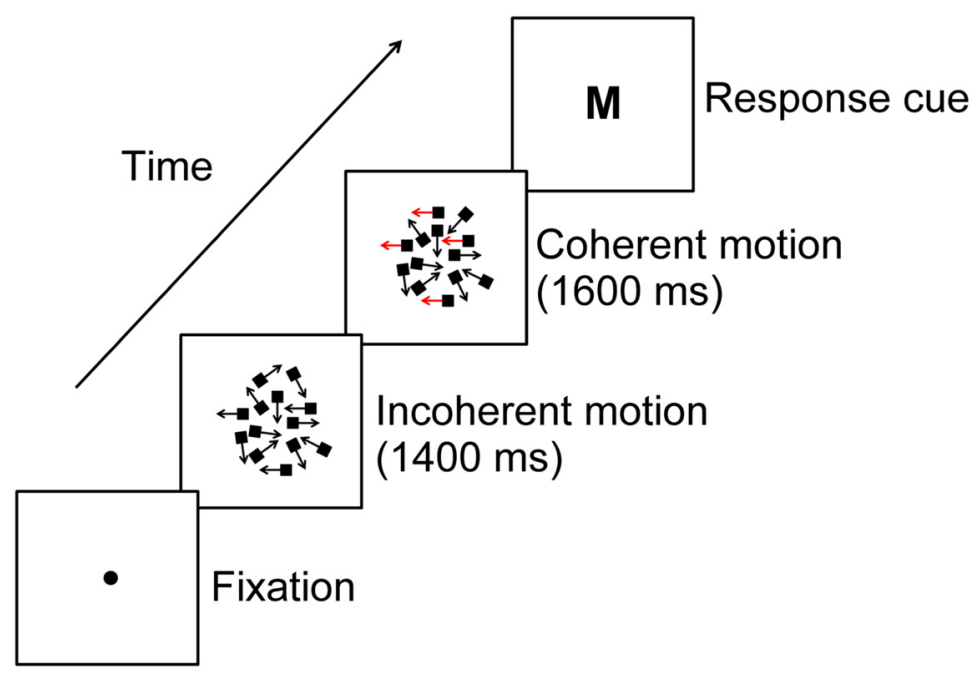

Figure 1. Schematic of the delayed response random dot motion task. In the No S-E condition, participants were instructed to ignore the kinetogram and make a speeded left or right hand button press solely on the basis of the response cue. In the Varied S-E condition, the stimulus-response association was withheld until the appearance of the response cue. An $\mathrm{M}$ cue indicated that leftward motion should be reported with a left hand press and rightward motion with a right hand press, whereas an $S$ cue indicated the reverse mapping (i.e., a left hand press for rightward motion and a right hand press for leftward motion). In the Fixed $S$-E condition, participants always indicated leftward motion with a left hand press and rightward motion with a right hand press, and the cue simply indicated the number of presses required (M, 1 press; $S, 2$ presses).

ology studies have indicated that such build-to-threshold "decision variable" signals may fall into at least two categories: effector-selective signals, which track decision formation when the decision entails a specific action (e.g., limb-selective betaband activity) (Donner et al., 2009; O'Connell et al., 2012; de Lange et al., 2013); and a domain-general signal, which represents decisions independently of specific sensory or motor requirements (the centro-parietal positivity [CPP]) (O'Connell et al., 2012; Kelly and O'Connell, 2013; Twomey et al., 2015). Although it has been verified that these signal types differ in the most obvious way, in that effector-selective preparation signals are absent when no responses are required while the CPP remains (O'Connell et al., 2012), much is yet to be elaborated on their functional distinctions.

A notable feature of effector-selective signals in single-unit and noninvasive recordings is that they maintain an elevated level of activity during the interval between commitment and action when the decision report must be delayed until the appearance of a response cue (Shadlen and Newsome, 2001; Donner et al., 2009). This observation has directly informed the model assumptions used in many functional neuroimaging studies to isolate domain-general decision regions (Heekeren et al., 2004, 2006; Pleger et al., 2006). However, it has yet to be determined whether the CPP exhibits the same properties. Maintaining a sustained level of motor preparation would facilitate the rapid execution of the decision-reporting action, but the benefits of sustainment for a domain-general decision process are less clear. One possibility is that sustainment of domain-general decision signals may only be beneficial when a choice cannot be immediately mapped to a specific action, such as when participants must await a response cue to learn the appropriate stimulusresponse association.

The present study sought to address these questions using a delayed-response variant of a prototypical perceptual discrimination task that participants performed with and without foreknowledge of the correct decision reporting action. In so doing, we reveal that domain-general and effector-selective decision sig- nals exhibit qualitatively different dynamics during the interval between choice and action.

\section{Materials and Methods \\ Participants. Twenty-five participants took part in the study. Two participants were ex- cluded due to excessive artifacts in their EEG data ( $<50 \%$ trials remaining), yielding a final sample of 23 participants $(9$ male, 1 left- handed), aged $24.6 \pm 6.2$ years (mean $\pm \mathrm{SD}$ ). All participants had normal or corrected-to- normal vision, no history of psychiatric illness or head injury and provided written informed consent in advance of testing. All procedures were approved by the Trinity College Dublin ethics committee in accordance with the Dec- laration of Helsinki.}

Random dot motion paradigm. We used a discrete-trial version of a flickered motion discrimination task modeled on the classic random dot motion task (Newsome et al., 1989; Britten et al., 1992; Kelly and O'Connell, 2013) in which participants judged the direction of motion in a patch of moving dots (Fig. 1). Participants made a simultaneous left and right mouse button click to initiate each trial upon which the dot kinetogram appeared. Initially, the dots moved incoherently for a period of $1400 \mathrm{~ms}$ after which they underwent an instantaneous transition to either leftward or rightward coherent motion. The stimulus disappeared after a further $1600 \mathrm{~ms}$ and was replaced by a response cue. The initial period of incoherent motion was introduced to ensure that sensory potentials evoked by stimulus onset would not impinge on activity associated with the decision-making process. Participants were made aware of this feature and informed that coherent motion would appear after $1400 \mathrm{~ms}$ on every trial. Motion direction and coherence (20\% or $30 \%)$ were varied independently and randomly across trials. The response cue always took the form of a centrally presented "S" or "M" with font size of $5 \mathrm{~mm}$. Participants performed the task under three experimental conditions in which we manipulated the stimulus-effector (S-E) mapping.

In the first condition (No S-E), coherent motion was incidental to the requirements of the task. Participants were instructed to maintain central fixation, ignore the kinetogram, and make a speeded left or right hand button press solely on the basis of the response cue, where $\mathrm{M}$ indicated a left press and $\mathrm{S}$ a right press. In the two remaining conditions, participants were required to judge the direction of coherent motion, and we manipulated whether the evolving perceptual decision could be directly translated into a specific motor plan. In the Varied S-E condition, the stimulus-response association was withheld from the participant until the appearance of the response cue. An M cue indicated that leftward motion should be reported with a left hand press and rightward motion with a right hand press, whereas an $\mathrm{S}$ cue indicated the reverse mapping (i.e., a left hand press for rightward motion and a right hand press for leftward motion). In the fixed S-E mapping condition, participants always indicated leftward motion with a left hand press and rightward motion with a right hand press and the cue simply indicated the number of presses required ( $M, 1$ press; $S$, 2 presses). This variation in the number of required button clicks was introduced to ensure that participants would be obliged to determine the identity of the response cue in all conditions before responding. In all conditions, participants were advised to respond as quickly as possible once the response cue appeared, with the trial ending as soon as the press was made. If the press was made before the onset of the response, cue participants were informed that it was "Too Fast"; however, if it was made $>1200 \mathrm{~ms}$ following the appearance of the response cue they were informed that it was "Too Slow." At the end of each trial, participants were advised as to whether their decision report was "Correct," "Wrong," or "Too Slow." Addi- 
tional feedback pertaining to the number of trials responded to correctly of a maximum of 30 was provided at the end of each block to promote effort maintenance.

Testing took place in a dark, sound-attenuated room, with participants seated at a distance of $\sim 50 \mathrm{~cm}$ from the visual display. Participants always completed the No S-E condition first to ensure that they were unaware of the presence of coherent motion, and this was confirmed at follow-up. The Fixed and Varied S-E mapping conditions were completed in counterbalanced order. Each participant completed 5 blocks of 30 trials per condition. Visual stimuli were presented against a black background on a $51 \mathrm{~cm}$ CRT monitor with a refresh rate of $85 \mathrm{~Hz}$ and a resolution of $1024 \times 768 \mathrm{~Hz}$. Participants were instructed to fixate on a centrally presented $5 \times 5$ pixel white point for the duration of the task in all conditions. The random dot motion stimulus was presented within an 8 degree aperture centered on fixation. During incoherent motion, an average of 118, $6 \times 6$ pixel white dots were placed randomly and independently within the circular aperture on each of a sequence of $58.8 \mathrm{~ms}$ frames played at 17 frames per second. This resulted in an on-off flicker at the same rate, giving rise to a steady-state visual-evoked potential (SSVEP) at $17 \mathrm{~Hz}$ in the EEG. During coherent motion, a proportion of the dots were randomly selected on each frame to be displaced by a fixed distance of 0.353 degrees in either the leftward or rightward direction on the following frame, resulting in a motion speed of 6 degrees per second.

EEG acquisition and preprocessing. Continuous EEG data were acquired using an ActiveTwo system (BioSemi) from 128 scalp electrodes and digitized at $512 \mathrm{~Hz}$. Vertical and horizontal eye movements were recorded using two vertical electro-oculogram (EOG) electrodes placed above and below the left eye and two horizontal electrodes placed at the outer canthus of each eye, respectively. Data were analyzed using custom scripts in MATLAB (The MathWorks), drawing on EEGLAB (Delorme and Makeig, 2004) routines for reading in data files and spherical spline interpolation of noisy channels. The continuous recordings at all channels were detrended to minimize the influence of slow drifts and were low-pass filtered $<35 \mathrm{~Hz}$ using a Hamming-windowed sinc finite impulse response (FIR) filter. For all of the principal analyses, the EEG data were segmented into epochs of -412 to $1700 \mathrm{~ms}$. The epochs were baseline-corrected relative to the $117 \mathrm{~ms}$ (two SSVEP cycles) interval preceding coherent motion onset. Trials were rejected if the bipolar vertical EOG signal (upper minus lower) exceeded an absolute value of 200 $\mu \mathrm{V}$ or if any scalp channel exceeded $100 \mu \mathrm{V}$ at any time during the epoch. Response-aligned traces were also extracted in the period spanning from -400 to $100 \mathrm{~ms}$ relative to response execution and any trials with artifacts within this time-range were excluded from this analysis. To remove the steady-state signal evoked by the on-off flicker of the stimulus, which was irrelevant to the present study, a $17 \mathrm{~Hz}$ notch filter was applied to the segmented EEG data for the ERP analysis. The filter was applied using a zero phase-shift Butterworth infinite impulse response (IIR) filter with a passband $<16 \mathrm{~Hz}$ and $-60 \mathrm{~dB}$ response at $16.5 \mathrm{~Hz}$, and a passband $>18$ $\mathrm{Hz}$ and $-60 \mathrm{~dB}$ response at $17.5 \mathrm{~Hz}$. The single-trial EEG data were converted to current source density (Kayser and Tenke, 2006) to increase spatial selectivity and to reduce the spatial blurring effects of volume conduction. This transformation was applied to minimize the projection of a negativity at frontocentral electrodes to the more posterior centroparietal electrodes (Kelly and O'Connell, 2013; Twomey et al., 2015). Because the signal topography, onset latency, build-up rate, peak amplitude, and latency of the signal did not differ for leftward versus rightward motion (all $p>0.05$ ); we collapsed across motion direction in all CPP analyses.

Measurement of electrophysiological decision signals. The domaingeneral CPP was measured at a single centroparietal electrode centered on the region of maximum component amplitude identified in the grand-average stimulus-aligned topography collapsing the Fixed and Varied S-E mapping conditions (Fig. 2C). As we had a strong a priori expectation that the CPP would be absent in the No S-E mapping condition based on previous findings (O'Connell et al., 2012), these trials were excluded from the topography for the purposes of channel selection. The CPP bears many parameters that impact on the timing and accuracy of decisions (O'Connell et al., 2012; Kelly and O'Connell, 2013), including onset latency, rate of rise, peak amplitude, and peak latency, which have been posited to index the start time and rate of evidence accumulation and the level and timing of its cessation, respectively (Kelly and O'Connell, 2015).

CPP onset latency was calculated using the Schwarzenau et al. (1998) regression-based method, which defines signal onset as the "breakpoint" between two intersecting lines that are fit to the ERP waveform, one fitted to the baseline and another to the rising slope of the ERP (for a similar approach, see Cespón et al., 2013). This method was applied separately to each participant's average stimulus-aligned waveform that was denoised by low-pass filtering the data $<6 \mathrm{~Hz}$ using a zero phase-shift, fourthorder Butterworth IIR filter (Spencer, 2004). The rate of rise of the stimulus-aligned CPP was measured as the slope of a straight line fitted to the unfiltered ERP waveform, using the interval 100 to $400 \mathrm{~ms}$ postevidence onset. Average stimulus- and response-aligned CPP peak amplitude per participant was measured as the maximum trial-average signal amplitude within windows of 350 to $600 \mathrm{~ms}$ and -100 to $100 \mathrm{~ms}$ relative to evidence-onset and response execution on each trial, respectively. Peak latency was measured as the time at which this maximum signal amplitude occurred in the same interval in the averaged trace of each condition and subject. Topographical differences in the CPP within a time window of 350 to $600 \mathrm{~ms}$ postevidence onset, on left and right motion trials and in the Fixed and Varied S-E mapping conditions were assessed using a nonparametric randomization test known as a paired topographic ANOVA (TANOVA) (Murray et al., 2008), implemented using Cartool software (http://www.fbmlab.com/cartool-software/). Specifically, a TANOVA tests for differences in global dissimilarity of EEG activity between two conditions by assessing if the topographies are significantly different from each other on a time point-by-time point basis (Murray et al., 2008). Finally, we measured the area under the curve of each participant's stimulus-aligned CPP waveforms, collapsed across the Fixed and Varied S-E mapping conditions, using the trapezoid method with unit spacing in MATLAB (The MathWorks). This additional measure was included to generate predictions for fMRI research because the BOLD response is assumed to scale with the integrated neural activity for a given time interval (Kayser et al., 2010).

Power estimates for all $\beta$ analyses were calculated using the Fast Fourier Transform, integrating across the $8-30 \mathrm{~Hz}$ range but excluding the single frequency bin at precisely $17 \mathrm{~Hz}$ (stimulus flicker frequency). To ensure that SSVEP power was fully contained in this omitted frequency bin, we used data segments of exactly 7 cycles of the $17 \mathrm{~Hz}$ SSVEP (i.e., $412 \mathrm{~ms}$ ) with no tapering. $\beta$ power was thus measured in each of a series of time segments taken in $58.82 \mathrm{~ms}$ steps, comprising a Short Time Fourier Transform. To identify channels exhibiting the strongest effector-selective activity, we generated a grand-average topography from the interval $-200 \mathrm{~ms}$ to 0 with respect to response, with right button press trials subtracted from left button press trials collapsed across all conditions (Fig. 2A). Based on inspection of this topography, we chose the standard left- and right-hemisphere motor sites $\mathrm{C} 3$ and $\mathrm{C} 4$ for $\beta$-band amplitude measurements. Stimulus- and response-aligned $\beta$-band lateralization was calculated by subtracting amplitude values at the electrode ipsilateral to the responding hand from those at the electrode contralateral to the responding hand (contralateral - ipsilateral). Thus, more negative $\beta$ lateralization values were indicative of greater preparation of the responding hand. The impact of motion coherence on the build-up rate of the stimulus-aligned $\beta$-trace was measured in the Fixed S-E mapping condition as the slope of a straight line fitted to the lateralization time course, using the interval 200 to $500 \mathrm{~ms}$ post-evidence onset.

A single-trial surface plot was generated to examine the single-trial dynamics of the CPP (curved black line). For this analysis, the stimulusaligned CPP waveforms were denoised by low-pass filtering the data $<6$ $\mathrm{Hz}$ using a zero phase-shift fourth-order Butterworth IIR filter (Spencer, 2004) and were pooled across S-E mapping condition (Fixed and Varied), motion direction, coherence, and participants. The CPP waveforms were subsequently sorted according to the signal's single-trial peak latency within a window of 200 to $1600 \mathrm{~ms}$ post-evidence onset and were smoothed over bins of 100 trials with a Gaussian-weighted moving average (Fig. 2E). 
A

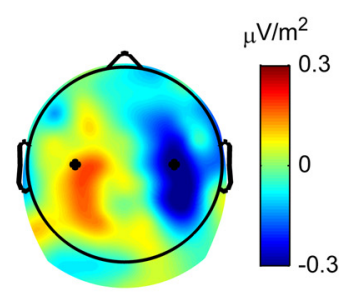

C

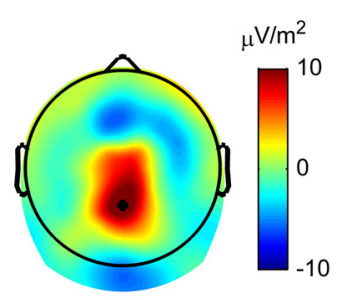

B Coherent motion

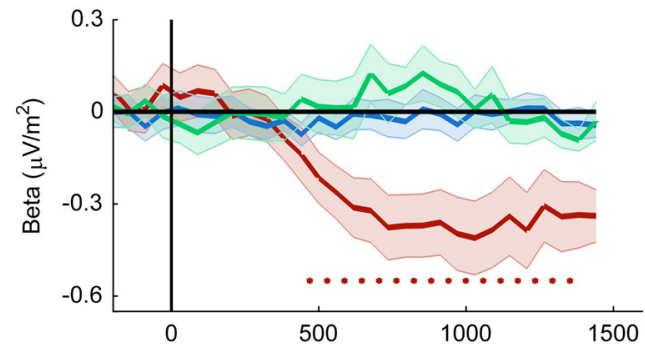

Cue

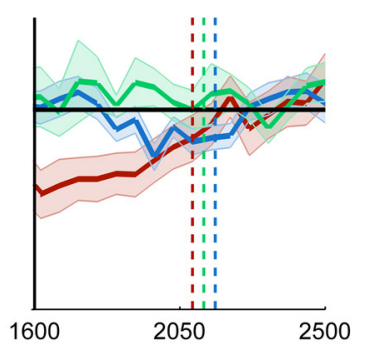

D

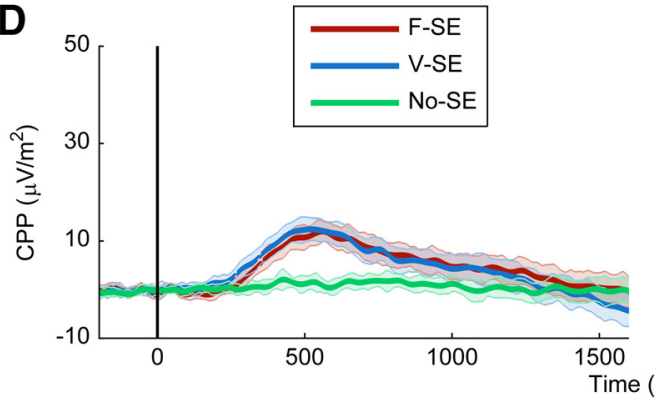

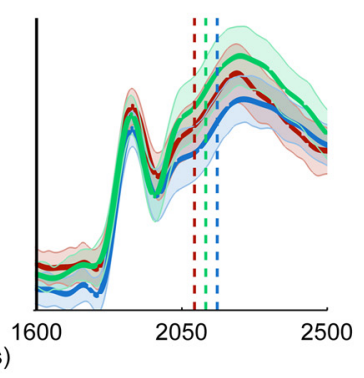

$\mu \mathrm{V} / \mathrm{m}^{2}$

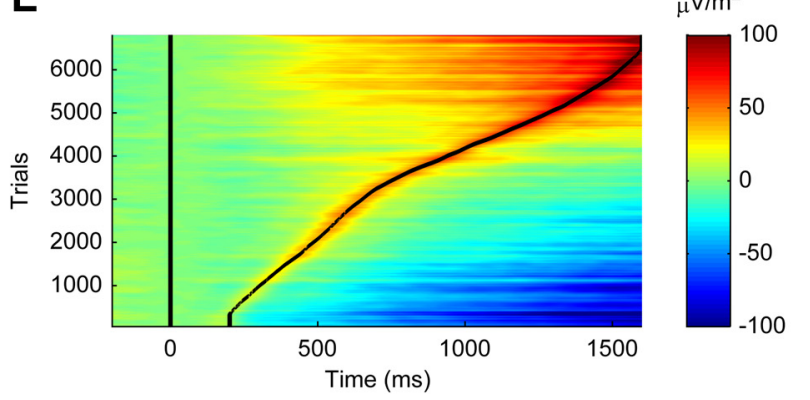

E
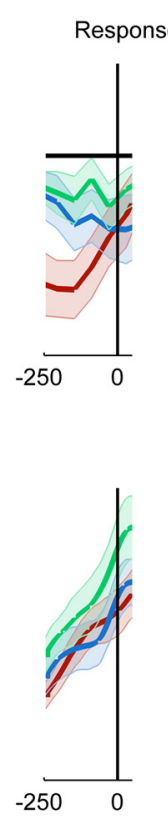

Figure 2. Impact of foreknowledge of stimulus-effector (S-E) mapping on abstract and effector-selective signatures of evidence accumulation in the human brain. $\boldsymbol{B}, \mathbf{D}$, Error bars indicate SEM across subjects. Color bar represents signal amplitude. The colored vertical lines indicate mean RT for each condition. $A$, Grand average signal topography of the difference between trials on which participants responded with a left versus right button click (collapsing across the three $S$-E mapping conditions) across a window of -200 to $0 \mathrm{~ms}$ centered on response execution. $\boldsymbol{B}, \beta$-band lateralization traces $(8-16$ and $18-30 \mathrm{~Hz})$ measured as the average difference in amplitude over motor regions contralateral minus ipsilateral to the responding hand, aligned to coherent motion onset (left), cue (middle), and response execution (right). Markers running along the bottom of the plot indicate the time points at which the lateralized $\beta$-band traces deviated significantly from zero $(p<0.05)$; a significant and sustained lateralization with respect to the hand of eventual response was only observed across the decision timeframe when the decision-reporting effector was known in advance. C, Grand-average signal scalp topography of the Fixed and Varied S-E mapping conditions across a time window of 400 to $550 \mathrm{~ms}$ centered on target onset. D, (PP waveforms aligned to the onset of coherent motion (left), cue (middle), and response execution (right). $\boldsymbol{E}$, Single-trial CPP surface plot sorted from the earliest to latest CPP peak latencies (curved black line; right) in the two motion discrimination conditions shows that the CPP did not remain at an elevated level after decision but descended toward baseline levels upon reaching its peak. Although there was considerable variability in the time at which the CPP reached its peak, it consistently returned to baseline levels shortly afterward. Although the abstract and effector-selective signals appear to accumulate sensory evidence in a similar way, they are dissociable in terms of their post-decision dynamics.

Statistical analyses. A one-way repeated-measures ANOVA was used to examine the impact of S-E mapping on reaction time (RT). As the data were not normally distributed, we measured the effect of this same manipulation on accuracy rates using three separate, nonparametric Wilcoxon signed-rank tests. The impact of coherence on accuracy and RT was measured using a Wilcoxon signed-rank test and a paired-samples $t$ test, respectively. As the CPP was absent in the No S-E condition, we used a series of paired-samples $t$ tests to explore the relationship between foreknowledge of S-E mapping and the onset latency, build-up rate, peak amplitude, and latency of the stimulus-aligned CPP in the two motion discrimination conditions (Fixed and Varied S-E). Two paired TANOVAs were used to identify possible topographical differences in the CPP as a function of motion direction and S-E mapping condition (Fixed and Varied). The impact of foreknowledge of S-E mapping on stimulus- and response-aligned effector-specific motor preparation signal was examined using two one-way repeated-measures ANOVAs. The effect of foreknowledge of S-E mapping on the peak amplitude of the response- aligned CPP was established using an additional one-way repeatedmeasures ANOVA.

To measure the impact of coherence on the build-up rate, peak amplitude, and area under the curve of the stimulus-aligned CPP in the average waveform collapsed across S-E mapping conditions (Fixed and Varied) and aligned to coherent motion onset, we used a series of paired-samples $t$ tests. Two additional paired-samples $t$ tests were used to determine the impact of coherence on the build-up rate and area under the curve of stimulus-aligned $\beta$-band lateralization in the Fixed S-E mapping condition. Finally, the impact of coherence on mean $\beta$-band lateralization and the peak amplitude of the responsealigned CPP at response execution was assessed using two pairedsamples $t$ tests. Where ANOVAs were used for statistical analyses and sphericity was violated in factors with two or more levels, the Greenhouse-Geisser or the Huynh-Feldt corrected degrees of freedom are reported (the correction applied was contingent on the de- 
gree of violation). False discovery rate corrections (Benjamini and Hochberg, 1995; Benjamini and Yekutieli, 2001) were applied to the time point-by-time point $t$ tests to correct for multiple comparisons. Outlying participants were excluded from specific analyses if their values within that analysis were a distance of $>3$ times the interquartile range or $>2.5$ SDs from the mean.

\section{Results}

\section{Behavioral performance}

RT varied significantly across the three experimental conditions (with respect to coherent motion onset: mean $\pm \mathrm{SD}$; Fixed S-E: $2095 \pm 65 \mathrm{~ms}$; Varied S-E: $2184 \pm 71 \mathrm{~ms}$; No S-E: $\left.2138 \pm 52 \mathrm{~ms} ; F_{(2,44)}=28.4, p=0.0001\right)$ such that participants responded significantly faster in the Fixed S-E condition relative to the Varied S-E $(p=0.0001)$ and No S-E mapping conditions $(p=0.001)$ and in the No S-E condition relative to Varied S-E condition ( $p=0.01)$. Accuracy levels did not vary across conditions (Fixed S-E: $95.9 \pm 3.9 \%$; Varied S-E: $95.1 \pm$ 4.4\%; No S-E: $97.4 \pm 2.4 \%$ ). There was no significant difference in accuracy rates between any pair of S-E conditions (all $p>0.1$ ). Participants were more accurate (Low: $94.4 \pm 4.8 \%$; High: $96.7 \pm 3.4 \% ; Z=-3.2, p=0.001)$ and had faster RTs (Low: $2152 \pm 65 \mathrm{~ms}$; High: $2126 \pm 62 \mathrm{~ms} ; t_{(22)}=6.6, p=$ $0.0001)$ when coherence was high relative to when it was low.

\section{Foreknowledge of stimulus-effector mapping differentially impacts upon abstract and effector-selective decision signals} To explore the influence that foreknowledge of S-E mapping has on neural signatures of decision formation, we collapsed each participant's trials across levels of coherence and plotted the average signal time courses aligned to the onset of coherent motion, the cue and response execution for each mapping condition separately (Fig. 2).

Consistent with observations of effector-selective decision neurons in the monkey and human brain (Fixed S-E) (Shadlen and Newsome, 2001; Donner et al., 2009; de Lange et al., 2013), limb-selective $\beta$-band activity exhibited a building lateralization upon evidence onset and then a sustained lateralization up until the appearance of the response cue when the decision-reporting effector was known in advance. A one-way repeated-measures ANOVA indicated a main effect of Condition $\left(F_{(2,42)}=4.9, p=\right.$ 0.01 ), with significantly more stimulus-aligned $\beta$-band lateralization in the Fixed relative to the Varied and No S-E mapping conditions (Fixed S-E vs Varied S-E: $p=0.01$; Fixed S-E vs No S-E: $p=0.03$; Varied S-E vs No S-E: $p=0.7$; Fig. $2 B)$. Running $t$ tests against zero spanning the interval from 0 to $1441 \mathrm{~ms}$ confirmed significant and sustained lateralization in the Fixed S-E condition only ( 16 of 24 time points, $p<0.05$; all $p>0.05$ for the Varied and No S-E conditions; Fig. 2B, left).

Coherent motion onset only elicited a CPP when it was relevant to the task at hand (i.e., Fixed and Varied S-E). A series of $t$ tests against zero spanning the interval from 0 to $1600 \mathrm{~ms}$ confirmed that the CPP was absent in the No S-E condition (all $p>$ 0.05; Fig. 2D, left) (O'Connell et al., 2012). In addition, we found that the stimulus-aligned CPP was entirely unaffected by the presence or absence of foreknowledge regarding the decision reporting effector with neither its topography, onset latency, build-up rate, peak amplitude, nor peak latency differing between the Fixed and Varied S-E mapping conditions $(p>0.1$; Fig. $2 D$, left). Inspection of the stimulus-aligned waveforms elicited in the two motion discrimination conditions (Fixed and Varied S-E) indicated that the CPP did not remain at an elevated level during the interval before the response cue but descended toward baseline levels upon reaching its peak. A single-trial surface plot (Fig. 2E), sorted as a function of single-trial CPP peak latency confirmed that the CPP returned toward baseline upon reaching its peak amplitude. This plot also highlights that the apparent slow return to baseline levels in the grand-average waveforms is largely the product of the temporal dispersion of CPP peaks, consistent with the variable decision times typically observed on random dot motion tasks. Analysis of the response-aligned waveforms confirmed that $\beta$-lateralization reached a comparable level before response execution $\left(F_{(2,42)}=0.82, p=0.45\right.$; Fig. $2 B$, right). The appearance of the response cue elicited an additional positive going signal over centro-parietal electrodes that likely reflects a second decision process for identification of the cue letter (Fig. 2D, middle). Analysis of this second signal, in a response-aligned average waveform, indicated that there were no differences in amplitude across S-E mapping conditions (Fig. $2 D$, right; $F_{(2,44)}=2.1, p=0.14$ ).

\section{Impact of evidence strength on abstract versus effector- selective decision signals}

To explore the influence that sensory evidence strength has on neural signatures of decision formation, we plotted the average signal time courses aligned to the onset of coherent motion, the cue and response execution for each level of coherence separately (Fig. 3). For each signal, we collapsed across conditions that showed equally strong build-up in the above analyses (CPP: Fixed and Varied S-E; $\beta$-lateralization: Fixed S-E only). In keeping with previous reports (Kelly and O'Connell, 2013), the build-up rate of the CPP increased in proportion to the strength of sensory evidence, such that stronger coherence was associated with a steeper rate of rise $\left(t_{(22)}=-3.97, p=0.00006\right)$. The amplitude of the coherent motion-aligned signal peak increased with coherence $\left(t_{(22)}=-2.5, p=0.02\right.$; Fig. $3 A$, left $)$, an effect that is consistent with the impact of evidence strength on the variability of decision times (Kelly and O'Connell, 2013; Twomey et al., 2015). That is, to the extent that the peak latency of the CPP marks the end of evidence accumulation, greater cross-trial variability in decision times for lower coherences would result in greater temporal jitter in peak latency and hence yield smaller grand average signal amplitudes (Luck, 2014).

Efforts to identify human brain regions that play a domaingeneral role in decision formation have frequently relied on assumptions regarding the impact that varying sensory evidence should have on the BOLD response in a decision-making region. One common prediction has been that the amplitude of the BOLD response in a decision region should distinguish between high and low evidence trials (Heekeren et al., 2004, 2006; Liu and Pleskac, 2011), although there has been disagreement regarding the direction of that relationship. Because the BOLD response is assumed to scale with the integrated neural activity for the corresponding time period (Kayser et al., 2010), we measured the area under the CPP curve over the interval spanning from 0 to 1600 ms. This analysis indicated no significant effect of coherence $\left(t_{(22)}=0.18, p=0.86\right)$.

In contrast to the CPP and one previous report using a RT version of the random dot paradigm (de Lange et al., 2013), the rate of $\beta$-band lateralization in the Fixed S-E mapping condition did not increase in proportion to the strength of sensory evidence $\left(t_{(21)}=-0.39, p=0.697\right.$; Fig. $\left.3 B\right)$. Analysis of the responsealigned waveforms revealed that the CPP and $\beta$-lateralization reached a fixed amplitude before response execution regardless of sensory evidence strength (CPP: $t_{(22)}=-0.4, p=0.97, \beta$-band lateralization: $t_{(21)}=-1.1, p=0.28$; see Figure $3 A, B$, right). 

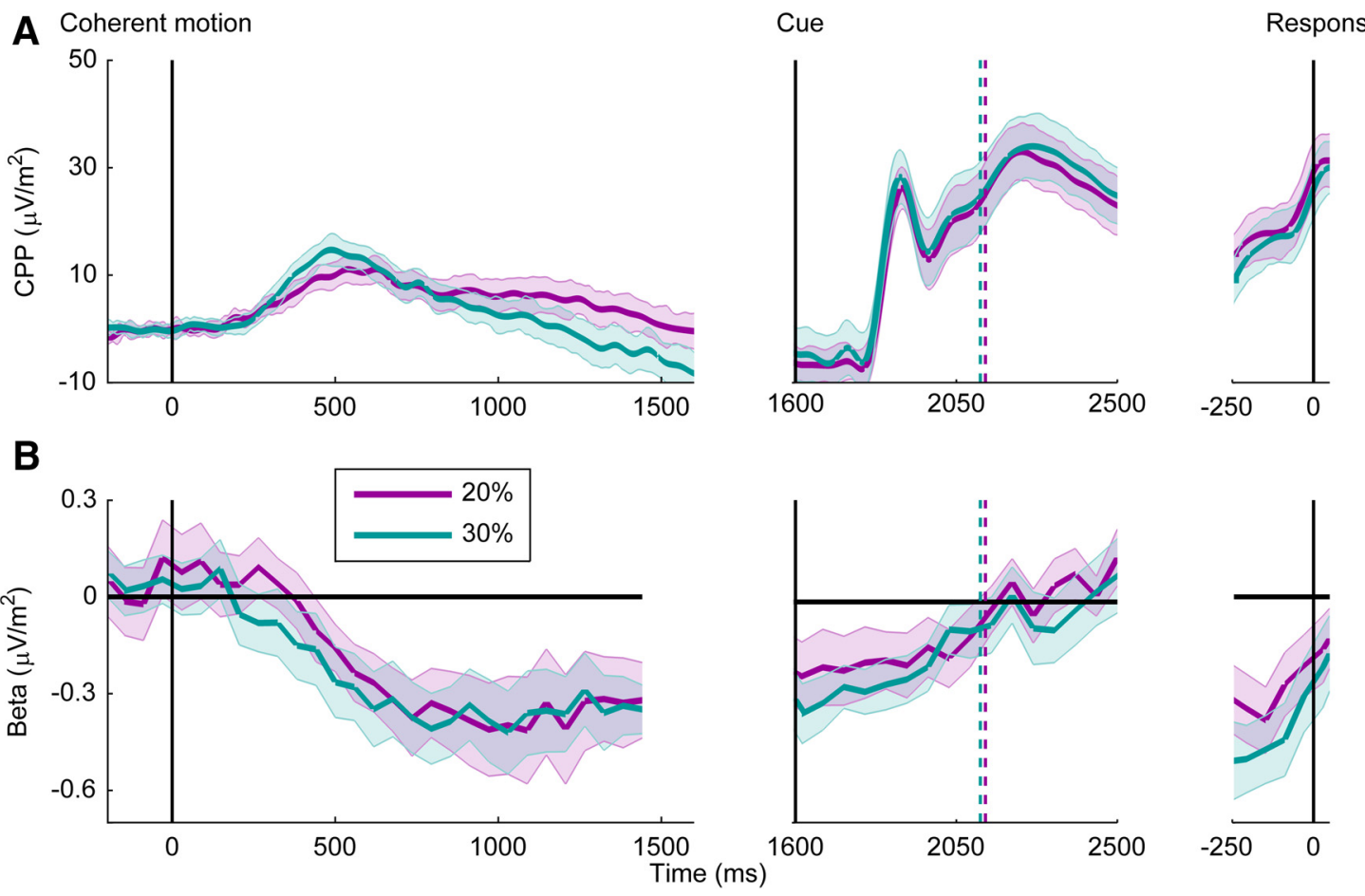

Figure 3. Decision variable signals as a function of coherence. Error bars indicate SEM across subjects. Colored vertical lines indicate mean RT for each coherence level. $A$, CPP waveforms collapsed across the motion discrimination conditions (Fixed and Varied S-E), aligned to coherent motion onset (left), cue (middle), and response execution (right) and plotted separately over time for the two coherence levels. $\boldsymbol{B}$, Lateralized $\beta$-band waveforms in the Fixed S-E mapping condition, aligned to coherent motion onset (left), cue (middle), and response execution (right) for both coherence levels.

\section{Discussion}

Our data indicate that effector-selective and domain-general decision signals exhibit qualitatively distinct dynamics in delayedresponse scenarios. Limb-selective $\beta$-band activity maintained a representation of the decision until the response cue appeared but only when the stimulus-effector association was known in advance. In contrast, the CPP was unaffected by such foreknowledge and, in all cases, returned toward baseline levels after reaching its peak. Our findings highlight the functional independence of abstract and effector-selective signatures of evidence accumulation and have important implications for neurophysiological and neuroimaging investigations of perceptual decision-making.

A number of single-unit neurophysiology studies have demonstrated that subpopulations of neurons within the lateral intraparietal area, continue to exhibit activity that discriminates the categorical alternatives even when the appropriate stimulusresponse mapping is withheld until a response cue (Freedman and Assad, 2006; Bennur and Gold, 2011) or when hand movements are used to report decisions instead of saccades (de Lafuente et al., 2015). In at least one study (Bennur and Gold, 2011), this category-selective activity appears to dissipate over time when the stimulus-response mapping is unknown, similar to the CPP under similar conditions. However, under conditions where the stimulus-response mapping is known in advance, lateral intraparietal area neurons coding for the saccade target location invariably do exhibit sustained activity up to the point of response execution; yet, the CPP returns to baseline under this condition in our data. Thus, the CPP's independence from the process of action preparation and execution appears to be a unique characteristic and highlights a key functional distinction from single-unit decision signals.

Efforts to isolate brain regions that are directly involved in decision formation from other task-activated structures using
fMRI have relied on assumptions that are inspired by observations from computational modeling and animal electrophysiology. One of the most common criteria for classifying decision structures has been a BOLD response that scales with evidence strength across trials (Heekeren et al., 2004, 2006; Pleger et al., 2006; Tosoni et al., 2008; Ho et al., 2009; Liu and Pleskac, 2011). The current study offers insight into the validity of these criteria as it used a delayed-response paradigm that is typical of those used in fMRI studies. Contrary to the evidence-scaling assumption, although the initial rate of rise of the CPP was sensitive to the strength of sensory evidence, its integrated activity across the trial duration was not (Heekeren et al., 2004, 2006; Pleger et al., 2006; Liu and Pleskac, 2011). Thus, to the extent that ERPs relate to BOLD activity, one implication of our findings is that contrasts based on these predictions will not necessarily produce differential activation of domain-general evidence accumulation regions when decision reports are withheld until the appearance of a response cue. In the present experiment, the influence of evidence strength on the build-up rate of the CPP was cancelled out in an integrated activity measure by its slow postpeak rate of decrease, although a larger discrepancy in build-up rates, driven by larger differences in coherence levels, would likely have yielded significant differences. In contrast, a well-established EEG index of motor preparation exhibited clear sustainment before the response cue when the stimulus-response mapping was known in advance. This result demonstrates that it cannot always be assumed that effector-selective and domain-general decision regions will exhibit the same dynamics across experimental conditions.

In contrast with previous reports (de Lange et al., 2013), $\beta$-band lateralization failed to show any modulation by evidence strength. This nonsignificant result should not be interpreted as evidence that such a relationship is entirely absent, particularly 
given the relatively small differences in coherence levels in this study. However, this observation is noteworthy in light of previous reports that the relationship between coherence and the slopes of lateral intraparietal area responses is diminished (Roitman and Shadlen, 2002) or absent (Rao et al., 2012) under delayed response conditions. It is plausible that delayed decision reporting significantly loosens the coupling between evidence integration and motor preparation, so that even dynamic dependencies as fundamental as that related to coherence fail to exhibit parallels across signal types. However, this issue will need to be addressed in a future, dedicated investigation.

While $\beta$-band activity maintained a representation of the chosen action during the interval between decision and response when the stimulus-effector mapping was known in advance, neither $\beta$ nor the CPP performed this role when the requisite effector could not be determined until presentation of the response cue. The question of how choice representations are actually maintained in delayed response scenarios will require further investigation to address (Shadlen and Kiani, 2013; Tsetsos et al., 2015).

Our results reveal fundamental functional dissociations between abstract and effector-selective evidence accumulation mechanisms and highlight the need for cross talk between electrophysiological and functional neuroimaging studies of perceptual decision-making.

\section{References}

Benjamini Y, Hochberg Y (1995) Controlling the false discovery rate: a practical and powerful approach to multiple testing. J R Stat Soc B 57:289-300.

Benjamini Y, Yekutieli D (2001) The control of the false discovery rate in multiple testing under dependency. Ann Stat 29:1165-1188. CrossRef

Bennur S, Gold JI (2011) Distinct representations of a perceptual decision and the associated oculomotor plan in the monkey lateral intraparietal area. J Neurosci 31:913-921. CrossRef Medline

Britten KH, Shadlen MN, Newsome WT, Movshon JA (1992) The analysis of visual motion: a comparison of neuronal and psychophysical performance. J Neurosci 12:4745-4765. Medline

Cespón J, Galdo-Álvarez S, Díaz F (2013) Age-related changes in ERP correlates of visuospatial and motor processes. Psychophysiology 50: 743-757. CrossRef Medline

de Lafuente V, Jazayeri M, Shadlen MN (2015) Representation of accumulating evidence for a decision in two parietal areas. J Neurosci 35:43064318. CrossRef Medline

de Lange FP, Rahnev DA, Donner TH, Lau H (2013) Prestimulus oscillatory activity over motor cortex reflects perceptual expectations. J Neurosci 33:1400-1410. CrossRef Medline

Delorme A, Makeig S (2004) EEGLAB: an open source toolbox for analysis of single-trial EEG dynamics including independent component analysis. J Neurosci Methods 134:9-21. CrossRef Medline

Donner TH, Siegel M, Fries P, Engel AK (2009) Buildup of choicepredictive activity in human motor cortex during perceptual decision making. Curr Biol 19:1581-1585. CrossRef Medline

Freedman DJ, Assad JA (2006) Experience-dependent representation of visual categories in parietal cortex. Nature 443:85-88. CrossRef Medline

Heekeren HR, Marrett S, Bandettini PA, Ungerleider LG (2004) A general mechanism for perceptual decision-making in the human brain. Nature 431:859-862. CrossRef Medline
Heekeren HR, Marrett S, Ruff DA, Bandettini PA, Ungerleider LG (2006) Involvement of human left dorsolateral prefrontal cortex in perceptual decision making is independent of response modality. Proc Natl Acad Sci U S A 103:10023-10028. CrossRef Medline

Ho TC, Brown S, Serences JT (2009) Domain general mechanisms of perceptual decision making in human cortex. J Neurosci 29:8675-8687. CrossRef Medline

Kayser AS, Buchsbaum BR, Erickson DT, D'Esposito M (2010) The functional anatomy of a perceptual decision in the human brain. J Neurophysiol 103:1179-1194. CrossRef Medline

Kayser J, Tenke CE (2006) Principal components analysis of Laplacian waveforms as a generic method for identifying ERP generator patterns: I. Evaluation with auditory oddball tasks. Clin Neurophysiol 117:348-368. CrossRef Medline

Kelly SP, O'Connell RG (2013) Internal and external influences on the rate of sensory evidence accumulation in the human brain. J Neurosci 33: 19434-19441. CrossRef Medline

Kelly SP, O'Connell RG (2015) The neural processes underlying perceptual decision making in humans: recent progress and future directions. J Physiol Paris 109:27-37. CrossRef Medline

Liu T, Pleskac TJ (2011) Neural correlates of evidence accumulation in a perceptual decision task. J Neurophysiol 106:2383-2398. CrossRef Medline

Luck SJ (2014) An introduction to the event-related potential technique. Cambridge, MA: Massachusetts Institute of Technology.

Murray MM, Brunet D, Michel CM (2008) Topographic ERP analyses: a step-by-step tutorial review. Brain Topogr 20:249-264. CrossRef Medline

Newsome WT, Britten KH, Movshon JA (1989) Neuronal correlates of a perceptual decision. Nature 341:52-54. CrossRef Medline

O'Connell RG, Dockree PM, Kelly SP (2012) A supramodal accumulationto-bound signal that determines perceptual decisions in humans. Nat Neurosci 15:1729-1735. CrossRef Medline

Pleger B, Ruff CC, Blankenburg F, Bestmann S, Wiech K, Stephan KE, Capilla A, Friston KJ, Dolan RJ (2006) Neural coding of tactile decisions in the human prefrontal cortex. J Neurosci 26:12596-12601. CrossRef Medline

Rao V, DeAngelis GC, Snyder LH (2012) Neural correlates of prior expectations of motion in the lateral intraparietal and middle temporal areas. J Neurosci 32:10063-10074. CrossRef Medline

Roitman JD, Shadlen MN (2002) Response of neurons in the lateral intraparietal area during a combined visual discrimination reaction time task. J Neurosci 22:9475-9489. Medline

Schwarzenau P, Falkenstein M, Hoormann J, Hohnsbein J (1998) A new method for the estimation of the onset of the lateralized readiness potential (LRP). Behav Res Methods Instrum Comput 30:110-117. CrossRef

Shadlen MN, Kiani R (2013) Decision making as a window on cognition. Neuron 80:791-806. CrossRef Medline

Shadlen MN, Newsome WT (2001) Neural basis of a perceptual decision in the parietal cortex (area LIP) of the rhesus monkey. J Neurophysiol 86: 1916-1936. Medline

Spencer KM (2004) Averaging, detection, and classification of single-trial ERPs: event-related potentials. In: Event-related potentials: a methods handbook (Handy TC, ed), pp. 209-228. Cambridge, MA: MIT Press.

Tosoni A, Galati G, Romani GL, Corbetta M (2008) Sensory-motor mechanisms in human parietal cortex underlie arbitrary visual decisions. Nat Neurosci 11:1446-1453. CrossRef Medline

Tsetsos K, Pfeffer T, Jentgens P, Donner TH (2015) Action planning and the timescale of evidence accumulation. PLoS One 10:e0129473. CrossRef Medline

Twomey DM, Murphy PR, Kelly SP, O'Connell RG (2015) The classic P300 encodes a build-to-threshold decision variable. Eur J Neurosci 42:1636-1643. CrossRef Medline 